\title{
Correction to: A New Lightweight Database Encryption and Security Scheme for Internet-of-Things
}

Jishun Liu, Yan Zhang, Zhengda Zhou, and Huabin Tang

\author{
Correction to: \\ Chapter "A New Lightweight Database Encryption \\ and Security Scheme for Internet-of-Things" \\ in: P. Qin et al. (Eds.): Data Science, CCIS 1258, \\ https://doi.org/10.1007/978-981-15-7984-4_13
}

The originally published version of the paper starting on p. 168 contained a data error. The figures in Table 1. on p. 173 have been corrected. 